\title{
Nuevos lineamientos de la Organización Panamericana de la Salud para la prevención y el control de la tuberculosis en los pueblos indígenas
}

New guidelines of the Pan American Health Organization for the prevention and control of tuberculosis in indigenous

people

\section{Comentado de:}

Lineamientos para la prevención y el control de la tuberculosis en los pueblos indígenas de la Región de las Américas. Washington, D.C.: Organización Panamericana de la Salud; 2020. Licencia: CC BY-NC-SA 3.0 IGO. ${ }^{1}$

\section{Introducción}

De acuerdo con información disponible de 17 países, la población indígena en las Américas alcanzaba en el año 2010 a 44,8 millones de habitantes distribuidos en 826 pueblos. En estas poblaciones, la tuberculosis sigue representando un problema de salud pública particularmente grave, con incidencias que pueden llegar a escalar hasta 75 veces la de la población general.

Desde la declaración de la epidemia de tuberculosis como emergencia de salud pública mundial por parte de la Organización Mundial de la Salud (OMS) en 1993, se han realizado diferentes avances, entre ellos la adopción en 2014 de la Estrategia Fin de la Tuberculosis ${ }^{2}$ (de aquí en adelante, la Estrategia) que busca poner fin a la epidemia para el año 2035.

Por su parte, luego de varias décadas de trabajo en intervenciones que incorporan un abordaje intercultural de la salud de los pueblos indígenas, la Organización Panamericana de la Salud (OPS) aprueba en 2017 la Política sobre etnicidad y salud ${ }^{3}$, documento mediante el cual la Región de las Américas se convierte en la primera región de la OMS en reconocer la importancia de adoptar un enfoque intercultural para abordar las inequidades en la salud.

Surge así el escenario propicio para la elaboración de estos nuevos lineamientos, que se organizan en dos grandes grupos: 1) los lineamientos generales para el abordaje intercultural de la tuberculosis, y; 2) los lineamientos para la implementación de la Estrategia.

Resaltaremos en este resumen los aspectos sobresalientes para cada grupo.

\section{¿A quiénes está dirigida esta guía?}

Los lineamientos del presente documento apuntan a brindar herramientas para abordar la problemática de la incidencia y transmisión de la tuberculosis en poblaciones vulnerables, como las indígenas, afrodescendientes, romaníes y otros grupos étnicos.

Entre los actores a quienes podrá resultar útil este documento se encuentran: autoridades y responsables gubernamentales del sector salud (en sus diferentes niveles), particularmente aquellas vinculadas con programas de manejo de la tuberculosis y/o asuntos indígenas o étnicos; líderes de asociaciones y organizaciones indígenas vinculados con la salud; personal de salud local que atiende a poblaciones indígenas; grupos de pacientes, organizaciones civiles y no gubernamentales; investigadores, docentes y potenciales donantes.

\section{Resumen de los lineamientos}

1. General para el abordaje intercultural de la tuberculosis

Como primer punto, resulta imprescindible analizar el comportamiento de la tuberculosis en los pueblos indígenas y la interacción de estos con los sistemas y servicios de salud.

Para ello es importante contar con datos nacionales y regionales que permitan comprender la distribución de la carga de enfermedad e identificar los recorridos que realizan los pacientes, la disponibilidad de recursos y las barreras existentes en el proceso de atención, desde la aparición de síntomas hasta que finaliza el tratamiento.

Se sugiere la conformación de un equipo intersectorial y multidisciplinario (ver sección: a quienes esta dirigida esta guía) cuyos integrantes cuenten con, o se estén formando para adquirir, habilidades y competencias en salud intercultural, que a su vez se organicen en grupos de trabajo para discutir, analizar y desagregar la información recolectada en aspectos clave, identificando el estado de situación, las fortalezas, debilidades y oportunidades de mejora.

Como segundo punto, se deberán reconocer los conocimientos y prácticas de las comunidades sobre la tuberculosis y elaborar rutas de atención intercultural.

Para ello se recomienda elaborar un directorio de actores clave (ej. líderes indígenas y no indígenas; representantes de la medicina tradicional y la medicina occidental, organizaciones no gubernamentales [ONG] y otras organizaciones), con quienes se puedan organizar encuentros para dialogar sobre los saberes, actitudes y prácticas de cada uno de los sectores en relación con la enfermedad y poder elaborar un resumen gráfico que permita luego rediseñar las rutas de atención, optimizar los recursos y priorizar las intervenciones.

\section{Enfocado en la implementación de la Estrategia}

Este lineamiento se desarrolla teniendo en cuenta tres pilares, que se resumen en la Figura 1.

\section{Pilar 1 - Atención y prevención integradas y centradas en el paciente}

Se hace hincapié en el fortalecimiento del diagnóstico rápido de la tuberculosis, valiéndose de pruebas de amplificación genética (por reacción en cadena de la polimerasa) como el Xpert ${ }^{\circledR}$ MTB/RIF y LF-LAM.

El primer método, consiste en la utilización de unidades portátiles, totalmente automatizadas, que permiten acelerar drásticamente (en menos de dos horas) la obtención de resultados a partir de las muestras estándar de secreciones respiratorias. Estos dispositivos requieren un mínimo de personal técnico e infraestructura y permiten ademas identificar las muestras resistentes a la rifampicina, que constituye un indicador fiable de diagnóstico de la tuberculosis multiresistente ${ }^{4}$. 
Figura 1. Pilares, principios y componentes de la Estrategia Fin del la Tuberculosis

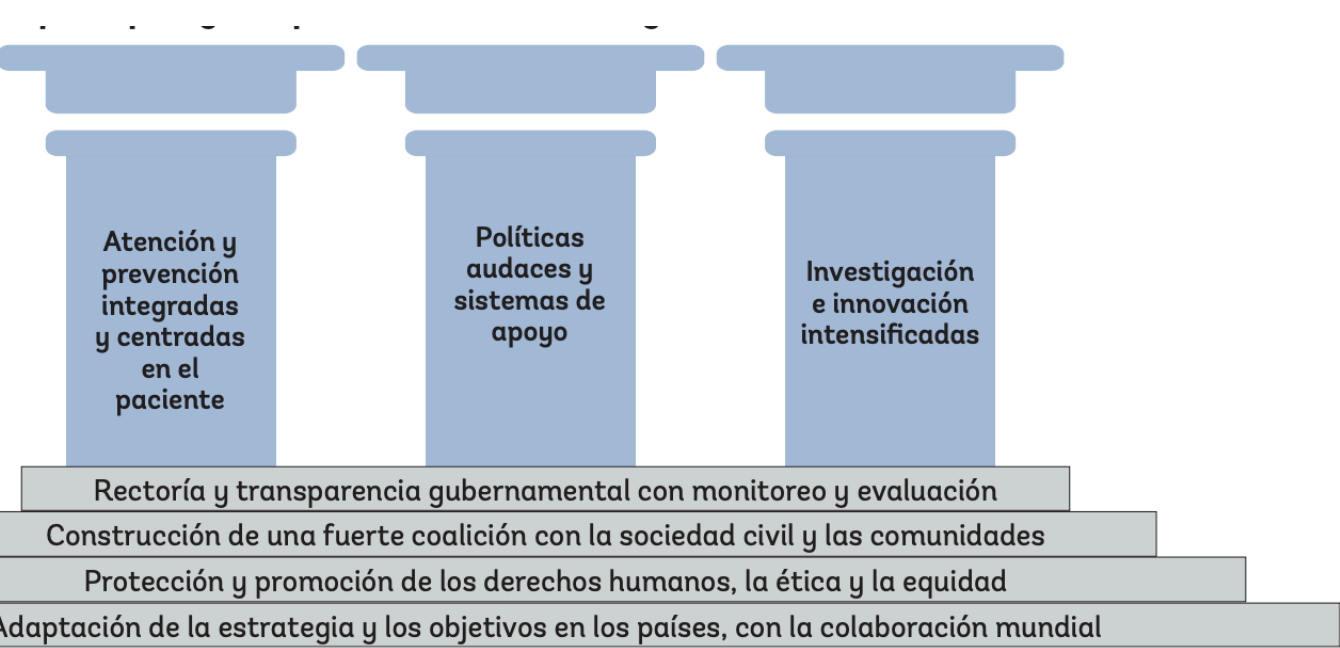

\section{Pilares y componentes}

1. Atención y prevención integrada de la tuberculosis centrada en el paciente

1.1. Diagnóstico, acceso universal a pruebas de sensibilidad a los medicamentos anti-TB y detección sistemática en grupos de alto riesgo

1.2. Tratamiento de las personas con tuberculosis y con tuberculosis farmacorresistente con apoyo centrado en el paciente

1.3. Actividades de colaboración TB/VIH y manejo de las comorbilidades

1.4. Tratamiento preventivo de las personas con alto riesgo y vacunación contra la tuberculosis

\section{Políticas audaces y sistemas de apoyo}

2.1. Compromiso político, con recursos suficientes para la atención a la tuberculosis y su prevención

2.2. Participación de las comunidades, las organizaciones de la sociedad civil y los prestadores de servicios de salud de los sectores público y privado

2.3. Política de cobertura universal de salud y marcos regulatorios para la notificación de los casos, los registros vitales, la calidad y el uso racional de los medicamentos y el control de infecciones

2.4. Protección social, reducción de la pobreza y las acciones sobre otros determinantes de la tuberculosis

\section{Investigación e innovación intensificada}

3.1. Descubrimiento, desarrollo y aplicación rápida de nuevas herramientas, intervenciones y estrategias

3.2. Investigación para optimizar la aplicación y el impacto de la Estrategia Fin de la TB y para promover las innovaciones

El segundo método, detección en orina del antígeno lipoarabinomanano micobacterial, está destinado a pacientes con síndrome de inmunodeficiencia adquirida $(\mathrm{VIH})$ y síntomas compatibles con tuberculosis. Si bien su sensibilidad es menor al Xpert ${ }^{\circledR}$ MTB/RIF, representa una alternativa cuando resulta dificultoso obtener muestras de esputo ${ }^{5}$.

Además, los sistemas de salud deberían poder hacer las estimaciones necesarias en cuanto a la logística de los recursos diagnósticos (laboratorios, equipamiento de rayos $\mathrm{X}$, etc.) para garantizar la recolección y el transporte a tiempo de las muestras, y ampliar el acceso y descentralización de las pruebas de diagnóstico molecular y sensibilidad a las drogas antituberculosas.

Con el recurso técnico disponible resulta importante entonces la realización sistemática de pruebas de detección de la tuberculosis en la población indígena. Para ello se deberá identificar y priorizar a las poblaciones de mayor riesgo: contactos domiciliarios, personas con VIH, personas con síntomas respiratorios crónicos (individuos con tos persistente de más de 15 días) personal de salud, niños de muy corta edad o desnutridos.

El tratamiento debe llegar de manera oportuna. Se deberá garantizar el acceso a los medicamentos anti-tuberculosos y realizar acciones de farmacovigilancia activa.

En las poblaciones indígenas resulta indispensable que los médicos tradicionales estén ampliamente vinculados con el apoyo al tratamiento y la adherencia de los pacientes, y que el personal de salud supervise el tratamiento bajo observación directa cuando sea necesario.

Se deberá asimismo realizar un abordaje de la coinfección VIH/tuberculosis desde la interculturalidad: garantizando la asesoría y el acceso a pruebas voluntarias de detección de VIH; promoviendo la integración de los servicios existentes de atención a la tuberculosis y VIH; garantizando el tratamiento oportuno de ambos y realizando el tamizaje de la tuberculosis en las personas con $\mathrm{VIH}$, ofreciendo un tratamiento preventivo en las personas con VIH en quienes de descarte una infección activa. 
Además, se deberán abordar otras comorbilidades y riesgos para la salud asociados a la tuberculosis, como p. ej.: la desnutrición, la diabetes, el consumo excesivo de alcohol y sustancias psicoactivas, el consumo de tabaco, la silicosis, los problemas de salud mental, la enfermedad pulmonar obstructiva crónica y otras enfermedades no transmisibles.

Por último, en este pilar se incluyen dos medidas adicionales de prevención: la vacunación con el bacilo de Calmettte-Guerín (BCG) y el tratamiento de la infección latente por tuberculosis.

La BCG forma parte habitual del esquema de vacunación infantil y ha demostrado prevenir formas graves de tuberculosis, como la meningitis tuberculosa y la tuberculosis miliar, ambas asociadas con una elevada mortalidad en menores de un año y en niños pequeños. En la población indígena en particular deberán observarse los factores que ponen en detrimento el acceso oportuno a la vacunación: el almacenamiento deficiente de vacunas, la pobre respuesta inmunológica por desnutrición, la disponibilidad de vacunas, los horarios de los centros de vacunación,y la identificación correcta de los niños ya vacunados, entre otros.

Las personas con infección latente comprobada por tuberculosis tienen un riesgo de enfermar del 5 a $10 \%$ a lo largo de la vida (riesgo que aumenta en los casos de coinfección con VIH), y en la mayoría de los casos esto ocurre dentro de los primeros cinco años.

Los principales grupos poblacionales a quienes se indica el tratamiento de la infección latente por tuberculosis, incluso sin requerir la prueba de la tuberculina, son las personas con VIH y los niños menores de cinco años que son contactos domiciliarios de un caso con tuberculosis activa. A los niños mayores de esa edad, los adolescentes y los adultos que son contactos de personas con tuberculosis pulmonar se recomienda administrar las pruebas y el tratamiento de la infección latente de forma sistemática.

\section{Pilar 2 - Políticas audaces y sistemas de apoyo}

Este pilar propone la incorporación de acciones estratégicas no solo del sector de la salud, sino de otros organismos, como los ministerios del interior (o aquel que lidere los temas de etnicidad y pueblos indígenas), de finanzas, justicia, trabajo, bienestar social, vivienda, minería y agricultura, entre otros; además de los socios no gubernamentales y la sociedad civil.

Resulta indispensable aquí el compromiso político, con recursos suficientes para la atención y la prevención de la tuberculosis. Para ello se requiere que en la actualización periódica del plan estratégico del Programa Nacional de la Tuberculosis en los países con población indígena se priorice la problemática de la tuberculosis en esta población. Se deberá garantizar que el plan estratégico nacional sea ambicioso e integral e incorpore presupuesto, ejecución, monitoreo y evaluación, investigación y asistencia técnica con un enfoque diferencial en las poblaciones indígenas y otras poblaciones vulnerables.

Asimismo, resulta importante identificar a los actores comunitarios con liderazgo y favorecer la participación de las comunidades, la organización de la sociedad civil y los prestadores de servicios de salud de los sectores público y privado, creando una fuerte coalición con las comunidades, las ONG y otras organizaciones de la sociedad civil. Se deberá involucrar también al sector privado y empresarial, aprovechando cuando sea oportuno la participación de empresas que desarrollan sus actividades en territorios ancestrales de los pueblos indígenas de las Américas.

Dentro de este pilar se hace también énfasis en continuar avanzando en las políticas de universalización de la salud, más aún cuando se trata de población indígena, entendiendo como tal al acceso y la utilización de los servicios de salud de buena calidad sin pasar por aprietos económicos para pagarlos; las mejoras en la notificación de los casos de tuberculosis; y la mejora en el proceso de producción de datos cuantitativos y cualitativos sobre la salud de la población.

Por último, se hace mención a la inclusión de las personas indígenas afectadas por la tuberculosis y a sus familias en programas de protección social.

\section{Pilar 3 - Investigación e innovación intensificadas}

Este pilar aborda la necesidad de encontrar nuevas y mejores herramientas para detectar, tratar y prevenir la enfermedad.

La OMS ha recomendado desde hace algunos años el tratamiento directamente observado (TDO) en la comunidad o en el hogar, con preferencia sobre el TDO en establecimientos de salud. Este ha demostrado tasas más altas de éxito del tratamiento y un número mayor de pacientes con negativización del esputo durante los primeros dos meses de poliquimioterapia.

Algunas intervenciones, como los recordatorios con SMS y el tratamiento observado por video, están bien posicionadas para brindar apoyo diario al tratamiento de la tuberculosis a gran escala.

La OMS, en alianza con otras organizaciones, elaboró un documento específico para dar claridad a las prioridades que la investigación operativa debe abordar para mejorar el cuidado y el control de la tuberculosis. Para ello es relevante promover la investigación y generar conocimientos sobre la diversidad de los conceptos de la salud y las enfermedades propias de los pueblos indígenas.

\section{Comentario}

Según informes de la Comisión Económica para América Latina y el Caribe, los pueblos indígenas presentan mayores niveles de pobreza y de falta de acceso a servicios básicos como el agua y el saneamiento, que influyen negativamente en su salud, siendo estos unos de los determinantes que explican la mayor incidencia de tuberculosis en comparación con la población general ${ }^{6}$. El surgimiento de los nuevos lineamientos de la OPS para la prevención y el control de la tuberculosis en los pueblos indígenas ${ }^{1}$, intenta ser una respuesta a esta problemática al brindar herramientas concretas para abordar la incidencia y transmisión de esta enfermedad con un enfoque intercultural. Del mismo se destaca la importancia del abordaje integral de esta patología, considerando todos los determinantes involucrados y la necesidad de desarrollar la investigación para tener datos epidemiológicos certeros de la verdadera carga de enfermedad en los pueblos indígenas, que permitan comprender las limitaciones y las posibilidades de mejora del proceso de atención, para así poder adecuarlo a las necesidades de la población.

En el 2017 se creó en el Instituto Universitario del Hospital Italiano de Buenos Aires (IUHIBA), en colaboración con el Hospital Italiano de Buenos Aires (HIBA), un proyecto socio-sanitario-educativo, con el objetivo de mejorar el acceso a la salud de la población de Santa Victoria Este, provincia de Salta (Figura 2 y $3,{ }^{7,8}$ ). Esta localidad pertenece al departamento de Rivadavia, el cual se ubica tercero a nivel nacional entre los departamentos con tasas más altas de notificación de tuberculosis ${ }^{9,10}$. 
Además, cuenta con un alto porcentaje de población indigena, en su mayoría pertenecientes a comunidades wichí, chorotes, tobas y chulupíes, en donde la tuberculosis, a pesar de ser una enfermedad prevenible y curable, continúa siendo uno de los principales problemas de salud pública.

Cabe agregar que en Santa Victoria Este, además de la alta incidencia de tuberculosis, coexisten otros problemas de salud que perpetúan y agravan su curso, como la desnutrición materno-infantil, el bajo porcentaje de cobertura de vacunación, el consumo problemático de sustancias y el inadecuado control de las enfermedades crónicas. A estas problemáticas se suman las dificultades en el acceso al sistema de salud por las grandes distancias entre las comunidades y los centros sanitarios, las inundaciones en épocas estivales, las diferencias culturales y religiosas, y la diversidad de idiomas y dialectos de la región. Luego de dos años de trabajo en terreno y un extenso análisis sobre las necesidades de la población, se inició dentro nuestro proyecto el Programa de Atención a los Pacientes con Tuberculosis con el propósito de evaluar, diagnosticar, tratar y realizar el seguimiento de las personas con esta patología, articulado con el Programa Nacional de Tuberculosis del Ministerio de Salud de la Nación Argentina. Más allá del compromiso con la comunidad, el programa busca capacitar y fortalecer al equipo de salud local, desde una perspectiva de respeto por la diversidad cultural. Como lo plantea la OMS en su trabajo sobre medicina tradicional $2014-2023^{11}$ y la OPS en el documento resumido ${ }^{1}$, incluir el modelo de la medicina tradicional, revalorizando los saberes ancestrales, reconociendo los diferentes conceptos de salud y las enfermedades propias de los pueblos originarios, resulta esencial para contribuir al acceso universal a la salud y es uno de los objetivos de nuestro proyecto.

Si bien la pobreza estructural, el acceso a la tecnología sanitaria necesaria para un diagnóstico rápido y la necesidad de personal técnico e infraestructura son uno de los puntos destacados en los nuevos lineamientos, en la actualidad en nuestro ámbito de trabajo, estos continúan constituyendo barreras para el manejo adecuado de esta problemática. Sin embargo, existen otros aspectos mencionados en este documento que se han logrado implementar en los últimos años en la región. De las propuestas enunciadas, observamos en nuestro trabajo en terreno la asistencia junto a efectores de salud locales para lograr un mayor acercamiento a las familias, el abordaje de otras condiciones de salud que perpetúan y agravan su curso como la desnutrición infantil, la realización de capacitaciones a personal de salud y a operadores territoriales, el establecimiento de vínculos y diálogos con representantes de la comunidad y equipo de salud local para adquirir habilidades que mejoren nuestras formas de atención, la participación de una mesa local intersectorial con el objetivo de trabajar de forma articulada e interdisciplinaria para responder a las demandas de la comunidad. Se destaca además el elevado porcentaje de cobertura de vacunación de BCG y acceso gratuito al tratamiento específico. En este marco de trabajo hemos tenido grandes logros, si bien los datos específicos respecto a la prevalencia de tuberculosis en la población de Santa Victoria Este se vieron sesgados por la pandemia, en nuestros primeros seis meses de implementación del Programa de tuberculosis se detectaron 28 casos índice, se evaluaron 207 contactos estrechos y se iniciaron 14 nuevos tratamientos.

\section{Conclusiones de los comentadores}

Creemos que la implementación de estos nuevos lineamientos es de suma importancia para mejorar la adherencia al tratamiento de tuberculosis. Como aspectos claves para continuar trabajando podríamos destacar: la necesidad de contar con datos sociosanitarios específicos sobre las comunidades originarias, la revalorización y la promoción de la medicina tradicional con la elaboración de circuitos de atención interculturales y la participación de equipos interdisciplinarios con representantes de las comunidades originarias en la planificación sanitaria.

Figura 2. Actividad asistencial a lactantes en el proyecto socio-sanitario-educativo del Instituto Universitario Hospital Italiano de Buenos Aires en Santa Victoria Este, Salta. Fuente: Facebook Instituto Universitario Hospital Italiano de Buenos Aires.

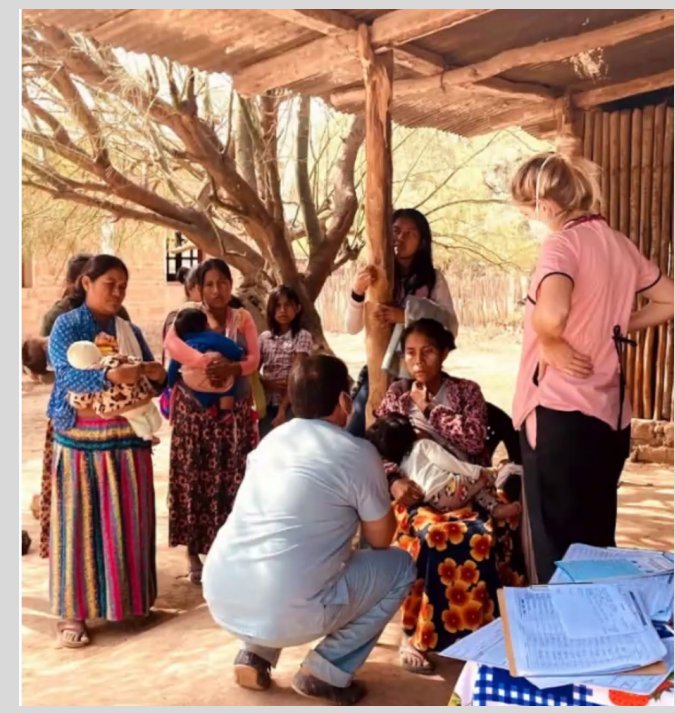


Figura 3. Actividades comunitarias en el proyecto socio-sanitario-educativo del Instituto Universitario Hospital Italiano de Buenos Aires en Santa Victoria Este, Salta. Fuente: Facebook Instituto Universitario Hospital Italiano de Buenos Aires.

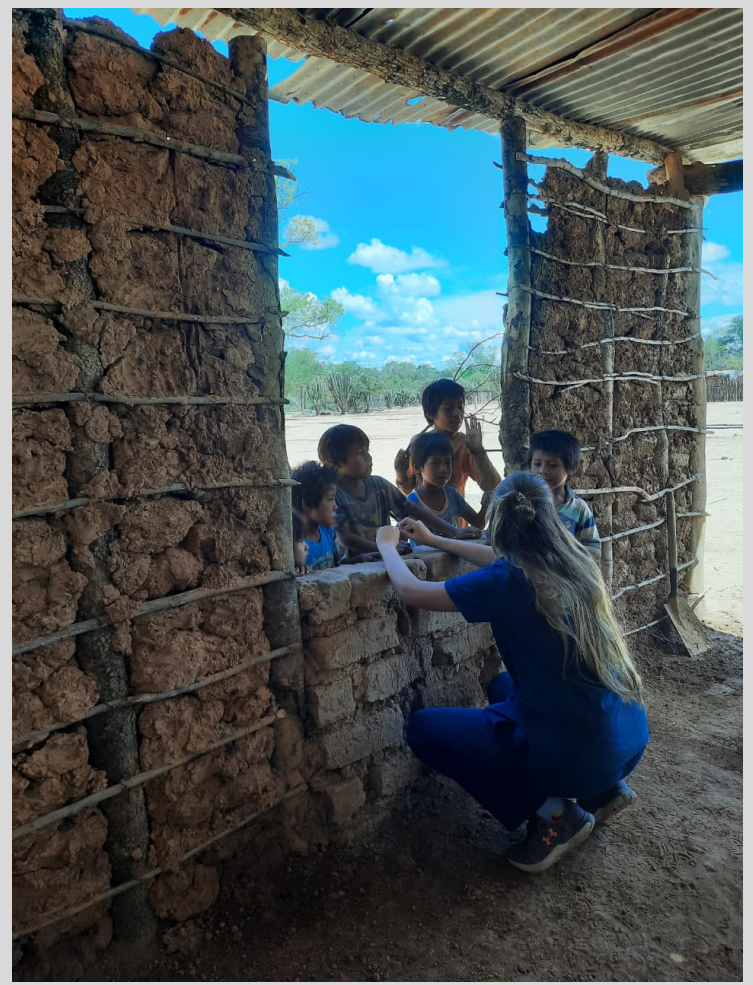

Isabel Bianchi Di Carcano [ Servicio de Clínica Pediátrica, Hospital Italiano de Buenos Aires. isabel.bianchi@hospitalitaliano.org.ar ]

Julieta Bitler [ Servicio de Medicina Familiar y Comunitaria, Hospital Italiano de Buenos Aires. julieta.bitler@hospitalitaliano.org.ar ]

Maria Rezzonico [ Servicio de Medicina Familiar y Comunitaria, Hospital Italiano de Buenos Aires. maria.rezzonico@hospitalitaliano.org.ar ]

Valentina Fernandez Alberdi [ Servicio de Clínica Pediátrica, Hospital Italiano de Buenos Aires. valentina.fernandez@hospitalitaliano.org.ar ]

Clara Lucía Torres Cabreros [ Servicio de Clínica Médica, Hospital Italiano de Buenos Aires. clara.torres@hospitalitaliano.org.ar ]

Gabriel Esteban Villalón [ Servicio de Medicina Familiar y Comunitaria, Hospital Italiano de Buenos Aires. gabriel.villalon@hospitalitaliano.org.ar ]

Bianchi Di Carcano L et al. Nuevos lineamientos de la Organización Panamericana de la Salud para la prevención y el control de la tuberculosis en los pueblos indígenas. Evid Actual Pract Ambul. 2021;24(3):e002140. Available from: https://dx.doi.org/10.51987/evidencia.v24i4.6926. Comentado de: Lineamientos para la prevención y el control de la tuberculosis en los pueblos indígenas de la Región de las Américas. Washington, D.C.: Organización Panamericana de la Salud; 2020. Licencia: CC BY-NC-SA 3.0 IGO.

\section{Referencias}

1. Organizacion Panamericana de la Salud. Lineamientos para la prevención y el control de la tuberculosis en los pueblos indígenas de la Región de las Américas. Washington, D.C: Organizacion Panamericana de la Salud; 2020. Licencia CC BY-NC-SA 3.0 IGO. Available from: https://iris.paho. org/bitstream/handle/10665.2/53308/9789275322772_spa.pdf?sequence=1\&isAllowed=y.

2. Organización Mundial De La Salud. Aplicación de la estrategia fin de la TB: aspectos esenciales. and others, editor. Ginebra: OMS; 2016. Available from: http://www.who.int/tb/publications/2015/end_tb_essential_spanish_web.pdf.

3. Comité Regional de la OMS para las Américas. Política sobre etnicidad y salud. In: 29.a Conferencia Sanitaria Panamericana, 25 al 29 de septiembre de 2017, Washington, D.C., EE.UU., Organización Panamericana de la Salud; 2017.Available from: https://www.paho.org/es/file/51612/download? token=_rOlHulh.

4. Sanchez JD. Manual de Capacitación en GeneXpert; 2017. Available from: https://www3.paho.org/hq/index.php?option=com content\&view=article\& id=12924: manual-de-capacitacion-en-genexpert\&ltemid=42250\&lang=es [Last access: 2021-08-07].

5. Bjerrum S, Schiller I, Dendukuri N, et al. Lateral flow urine lipoarabinomannan assay for detecting active tuberculosis in people living with HIV Cochrane Database Syst Rev. 2019;10(10):CD011420. Available from: 10.1002/14651858.CD011420.pub3. 
6. Comisión Económica para América Latina y el Caribe. Los pueblos indígenas en América Latina: avances en el último decenio y retos pendientes para la garantía de sus derechos. CEPAL; 2014.

7. Instituto Universitario Hospital Italiano de Buenos Aires. Proyecto Isthat Santa Victoria Este - IUHI; 2021. Available from: https://www.facebook.com/ pg/csuiuhi/posts/ [Last access: 2021-08-31]

8. Instituto Universitario Hospital Italiano de Buenos Aires. Compromiso social universitario; 2021. Available from: https://instituto.hospitalitaliano.org. ar/\#!/home/csu/inicio [Last access: 2021-08-31]

9. Bossio JC, Fernandez H, Arias S. Boletín sobre tuberculosis en la Argentina - Nro. 2. Programa Nacional de Control de Tuberculosis y Lepra. Ministerio de Salud y Desarrollo Social; 2019. Available from: https://bancos.salud.gob.ar/recurso/boletin-sobre-tuberculosis-en-argentina-no-2\#: : text=En\%20nuestro\%20pa\%C3\%ADs\%2C\%20la\%20TBC,A\%C3\%B10\%202019.

10. Bossio JC, Fernandez H, Arias S. Boletín sobre tuberculosis en la Argentina - Nro. 3. Programa Nacional de Control de Tuberculosis y Lepra. Ministerio de Salud y Desarrollo Social; 2020. Available from: https://bancos.salud.gob.ar/recurso/boletin-sobre-tuberculosis-en-argentina-no-3.

11. Organización Mundial de la Salud. Estrategia de la OMS sobre medicina tradicional 2014-2023. Ginebra: Organización Mundial de la Salud; 2013. Available from: https://apps.who.int/iris/handle/10665/95008. 